\title{
A Detailed Solution Framework for the Out-of-Plane Displacement of Circular Dielectric Elastomer Actuators
}

\author{
Hector Medina ${ }^{1}$, Carson Farmer ${ }^{2}$, and Daniel Korn ${ }^{2}$ \\ ${ }^{1}$ Liberty University \\ ${ }^{2}$ Affiliation not available
}

January 18, 2021

\begin{abstract}
The out-of-plane displacement (OPD) of a circular dielectric elastomer actuator (DEA) membrane has been explored in recent years for a variety of important applications. Circular DEAs consist of an elastomer membrane that is prestretched to a rigid frame and coated with compliant electrodes. Such a simple configuration has found many interesting applications such as in, pumps, pulse tracking, hopping search and rescue robots, dielectric elastomer (DE) generators for renewable energy harvesting, linear actuators, and many others. Here, we present an easy-to-follow implementation of the OPD equations for circular DEAs via a detailed numerical solution using the shooting method (SM) on a system of differential algebraic equations (DAE), based on previous theoretical work. The SM numerical solution to the system of DAEs is applied to a practical range of values based on the reported literature. Analysis of the results and comparison against other studies are provided. The current work provides a go-to framework for implementation in further research and development.
\end{abstract}

\section{Hosted file}

DetailedFramework for OPD for circular DEA_new.pdf available at https://authorea.com/users/ 390137/articles/504567-a-detailed-solution-framework-for-the-out-of-plane-displacementof-circular-dielectric-elastomer-actuators 